\title{
Utilization Gaps During the COVID-19 Pandemic: Racial and Ethnic Disparities in Telemedicine Uptake in Federally Qualified Health Center Clinics
}

\author{
Omolola E. Adepoju, PhD, MPH ${ }^{1,2}$ (D) , Minji Chae, $\mathrm{MS}^{2}$, \\ Chinedum O. Ojinnaka, MBBS, PhD, MPH ${ }^{3}$, Sharonya Shetty ${ }^{7}$, and Tracy Angelocci, $\mathrm{MD}^{4}$
}

'University of Houston, College of Medicine, 4849 Calhoun Road, Bldg 2, Houston, TX, USA; ${ }^{2}$ Humana Integrated Health Systems Sciences Institute, Houston, TX, USA; ${ }^{3}$ College of Health Solutions, Arizona State University, Phoenix, AZ, USA; “ ${ }^{2}$ Lone Star Circle of Care, Austin, TX, USA.

BACKGROUND: The Coronavirus Aid, Relief, and Economic Security (CARES) Act led to the rapid implementation of telemedicine across healthcare office settings. This innovation has the potential to improve healthcare use and ensure continuity of care. However, this delivery model could have an unintended consequence of worsening racial/ethnic disparities in healthcare utilization if adoption varies across sub-populations.

OBJECTIVE: To examine associations between telemedicine use and race/ethnicity between 3/1/2020 and 11/ $30 / 2020$, and the influence of other individual- and geographical-level factors on this relationship.

DESIGN: Cohort study

PARTICIPANTS: EMR data from 55 clinics in a FQHC network

MAIN MEASURES: The dependent variable was visit type (in-person vs. telemedicine). Predictors of interest were patient race and ethnicity. To account for repeated visits within each patient nested within clinic, a three-level, mixed-effects, multivariable, logistic regression model was used. Subgroup analyses examined correlates of telemedicine use in African American and Hispanic cohorts, separately.

KEY RESULTS: The analytic sample included 233,302 visits for 67,733 unique patients. African Americans (OR $=0.65,95 \%$ CI: 0.61, 0.69), Asians (OR = 0.58, 95\% CI: $0.52,0.65)$, and American Indians / Alaska Natives and other Pacific Islanders (OR $=0.82,95 \%$ CI: 0.70, 0.98) were significantly less likely to use telemedicine compared to Whites. Hispanics were also less likely to have a telemedicine visit (OR $=0.49,95 \%$ CI: $0.47,0.51)$ compared to non-Hispanics. Nonacute visits were more likely to be conducted via telemedicine. Distance to clinic exhibited a dose-response relationship such that patients who lived farthest from the clinics were most likely to have telemedicine visits. In the subgroup analyses to examine predictors of telemedicine use, the dose-response relationship between distance from clinic and telemedicine use persisted, with increasing distance associated with increasing likelihood of telemedicine use, in both African American and Hispanic cohorts. Nonacute visits were associated with telemedicine use in the Hispanic cohort, but not in the Black / African American cohort.

Received July 23, 2021

Accepted November 23, 2021

Published online February 2, 2022
CONCLUSION: Racial/ethnic disparities in telemedicine use persisted among this cohort. However, telemedicine improved utilization for African Americans and Hispanics living farther away from the clinic.

KEY WORDS: telemedicine; healthcare utilization; health disparities; FQHCs.

J Gen Intern Med 37(5):1191-7

DOI: $10.1007 / \mathrm{s} 11606-021-07304-4$

This is a U.S. government work and not under copyright protection in the U.S.; foreign copyright protection may apply 2021

\section{INTRODUCTION}

Access to healthcare is critical to maintaining health status and preventing disease. Unfortunately, many individuals still face obstacles in receiving such services, and health and health care disparities have continued to persist in the US. Despite the efforts of the Affordable Care Act to increase insurance coverage across the nation, racial and ethnic minority groups remain vulnerable and at higher risk for worse health outcomes. ${ }^{1-3}$ When compared to their White counterparts, Blacks / African Americans are 1.5 times more likely to be uninsured, and Hispanics are 2.5 times more likely to be uninsured. ${ }^{4}$ But ensuring access in a general sense is merely scratching the surface of a deeper and seemingly more intractable issue.

The emergence of the COVID-19 pandemic highlighted the need for affordable, digital, patient care options. Telemedicine, the delivery of clinical services through telephone and/orvideoconferencing tools, offers a potential method of bridging the gap in access to care while addressing health care demands during a global pandemic. Particularly, this novel model has been viewed as promising, especially in eliminating access-tocare issues for those living in underserved areas. ${ }^{5}$ Following the passage of the Coronavirus Aid, Relief, and Economic Security (CARES) Act in March $2020^{6}$, the Centers for Medicare \& Medicaid Services (CMS) lifted previously placed restrictions to allow health care office settings, including Federally Qualified Health Centers (FQHCs), to provide remote patient care through telemedicine. The rapid implementation of telemedicine across health care office settings has implications for racial/ ethnic disparities in health care utilization as it could reduce or 
eliminate barriers to health care use such as inadequate health care provider availability, lack of transportation, and the need to arrange for childcare or take time of work, thus ensuring appointment compliance. ${ }^{7,8}$ However, this delivery model could also have an unintended consequence of worsening racial/ethnic disparities in health care utilization if adoption varies across subpopulations.

Limited broadband access could limit patient use of health information technology. Hence, the gulf between those who have ready access to computers and internet and those who do not, known as the digital divide, could further advance health care disparities. For instance, only $66 \%$ of African American and $61 \%$ of Hispanic households have access to broadband compared to $79 \%$ of White households in the US. ${ }^{9}$ These disparities related to the digital divide have deepened among already-vulnerable populations with the onset of the COVID-19 pandemic. ${ }^{10}$ Although smartphones can also be used for telemedicine visitation, low-income individuals might not be able to afford the data services needed for such transaction. Other factors that could limit the adoption of telemedicine by patients include a preference for in-person visits and unsatisfactory patient experiences with telemedicine use. ${ }^{11}$ These limitations to telemedicine use have adverse implications for racial/ethnic minorities who are already at risk for suboptimal health care use and clinical outcomes.

This study analyzed the association between telemedicine use and race/ethnicity in FQHC clinics, as well as the influence of other individual- and geographical-level factors on this relationship. We also separately examined the determinants of telemedicine utilization in African American and Hispanic cohorts. Studies of this nature ensure that initiatives designed to improve access to care do not inadvertently perpetuate disparities across population groups. Understanding these relationships could guide interventions to increase telemedicine use among racial/ethnic minorities.

\section{METHODS}

\section{Data}

Visit-level data were pulled from electronic medical records (EMRs) at $23 \mathrm{FQHC}$ clinic locations (across 6 different counties and 55 individual clinics) in Texas. All patients were offered telemedicine at least once. For the purposes of this study, data on all patients who had one or more visits were pulled over a period of 9 months (from March 1, 2020 to November 30, 2020), reflecting the pattern of telemedicine visits in response to the COVID-19 pandemic at the time of this work. Data included patient demographic information, encounter-level information, and visit type (in-person or telemedicine).

\section{Measurement}

The dependent variable was visit type: in-person (0), telemedicine (1). Per clinic operations at these FQHC clinics, a telemedicine visit is a synchronous audiovisual interaction between the practitioner and the patient in separate locations. These clinics employed a telemedicine platform and technology support program beginning in mid-March 2020 (prior to this period, telemedicine visits consisted of $0.1 \%$ of all consults). The primary independent variable was patientreported race (White, Black / African American, Asian, American Indian / Alaska Native, mixed race) and ethnicity (nonHispanic, Hispanic). Other covariates included patient sociodemographic variables (age, gender), health insurance coverage, geographic factors (e.g., distance in miles to clinic, metropolitan/nonmetropolitan status, residence in a medically underserved area [MUA] as defined by the Health Resources $\&$ Services Administration (HRSA], acute-visit flag (acute vs. nonacute), and clinic characteristics (service lines offered, e.g., Family Practice, Mental Health, Obstetrics and Gynecology, Pediatrics, and Geriatrics). These variables have been previously found to be associated with health information technology use and health care utilization. ${ }^{7,8,10}$ In line with previous studies, missing datawere handled using listwise deletion. Only observations with data on all the variables of interest were included in the analyses. The final sample size was 233,302 visits for 67,733 unique patients within 55 clinics.

\section{Analysis}

Descriptive analyses employing frequencies and proportions were used to describe patient demographic characteristics. Chi-square tests were used to assess independent bivariate associations between patient characteristics and visit type, while multivariate analysis focused on adjusted associations between patient characteristics and visit type. In order to account for nested data structure (repeated visits within each patient nested within clinics), a three-level, mixed-effects, logistic regression model assessed the relationship between race/ethnicity and visit type, adjusting for patient sociodemographic characteristics and geographic classification. Patient project-assigned identification (ID) numbers and clinic location were included as separate random effects. We also separately stratified the analyses by race/ethnicity to examine correlates of telemedicine use in African American and Hispanics cohorts. This study was approved by an independent institutional review board in October 2020. All data management and analyses were performed using Stata 16.1. All statistical tests were two-sided, and findings were considered statistically significant at $p<0.05$.

\section{RESULTS}

\section{Sample Characteristics}

Overall, the sample represented 233,302 visits for 67,733 unique patients within 55 clinics. Over the 9-month study period, telemedicine visits constituted $32 \%$ of visits, while in-person visits accounted for the remaining $68 \%$. The sample 
comprised $48 \%$ Hispanics and 22\% racial minorities. Racial minority subgroups included Black / African American (15\%), Asian (3\%), American Indian / Alaska Native and other Pacific Islander (1.4\%), and mixed race (3\%). Children (0-18 years old) consisted $39 \%$ of the sample; older adults (65+ years old) consisted of 5\% of the sample, while nonelderly adults (18-64 years old) represented a majority of the sample at $56 \%$. Approximately $17 \%$ of the sample patients were privately insured, $6 \%$ were covered via Medicare, $43 \%$ were covered under Medicaid, and 33\% were uninsured. A majority of visits were nonacute $(71 \%)$. With regard to the geographic characteristics of patient residence, $96 \%$ resided in metropolitan areas and $50 \%$ were residents of MUAs. Over one-quarter of the sample lived within 5 miles of the clinic (27\%), $26 \%$ lived 5-10 miles away, 28\% lived 10-20 miles away, $16 \%$ lived 20-50 miles away, and 3\% lived more than 50 miles away (Table I).

\section{Telemedicine Utilization}

Bivariate associations between patient characteristics and visit type are shown in Table I. Telemedicine utilization varied by patient race (White at $24.0 \%$, Black / African American at 24.7\%, Asian at 23.7\%, American Indian / Alaska Native and other Pacific Islander at 20.3\%, and mixed race at 26\%, $p<$ 0.001 ) and ethnicity (Hispanic at $19.2 \%$ vs. non-Hispanic at $28.7 \%, p<0.001$ ). Telemedicine also varied by age such that older adults reported the highest telemedicine utilization (27.9\%) compared to nonelderly adults $(26.3 \%)$ and children (20.5\%). Patients enrolled in Medicare reported significantly higher telemedicine visits $(35.1 \%)$ compared to those who were Medicaid-insured (20.9\%), privately insured (30.2\%), and uninsured $(23.8 \% ; p<0.001)$. Telemedicine usage varied by metropolitan residence ( $21.2 \%$ nonmetropolitan vs. $24.3 \%$ metropolitan; $\mathrm{p}<0.001)$. Telemedicine use did not differ by residence in a MUA. Distance from clinic displayed a doseresponse relationship such that patients living farthest from the clinics were most likely to have telemedicine visits $(<5$ miles at $22.9 \%, 5-10$ miles at $23.5 \%, 10-20$ miles at $24.2 \%, 20-50$ miles at $25.4 \%$, and $>50$ miles at $34.9 \%, p<0.001$ ).

Table II shows the trends of telemedicine utilization by service line over the course of the 9-month study period (March-November 2020). Telemedicine was predominant in the Mental Health service line (highest at $70 \%$ in April) and less common in Obstetrics and Gynecology (highest at 6\% in May).

\section{Mixed Effects Regression Model}

Results from the mixed-effects regression model are presented in Table III. When compared to their White counterparts, Blacks / African Americans, Asians, and American Indians / Alaska Natives and other Pacific Islanders were all significantly less likely to have a telemedicine visit $(\mathrm{OR}=0.65, p<$ $0.001 ; \mathrm{OR}=0.58, p<0.001, \mathrm{OR}=0.82, p=0.03$, respectively). There was no statistically significant association between mixed race and telemedicine use. Compared to non-Hispanics, Hispanics were less likely to have a telemedicine visit (OR = $0.49, p<0.001)$.

Compared to females, males were less likely to have a telemedicine visit $(\mathrm{OR}=0.85, p<0.0014)$. Individuals younger than 18 years $(\mathrm{OR}=0.38, p<0.001)$ and older adults $(\mathrm{OR}$ $=0.82, p<0.001)$ were less likely to have a telemedicine visit when compared to nonelderly adults. Having an acute visit was associated with $11 \%$ lower odds of a telemedicine visit $(\mathrm{OR}=0.89, p<0.001)$ compared to a nonacute visit. Telemedicine use also varied by insurance coverage type. Compared to privately insured patients, Medicaid-insured patients $(\mathrm{OR}=0.76, p<0.001)$ and uninsured patients $(\mathrm{OR}=0.67, p<$ 0.001 ) were less likely to have a telemedicine visit.

Residence in a metropolitan area was associated with $17 \%$ higher odds of a telemedicine visit $(\mathrm{OR}=1.17, p=0.0231)$ compared to nonmetropolitan residence. Residence in an MUA was associated with $12 \%$ higher odds of a telemedicine visit $(\mathrm{OR}=1.12, p<0.001)$ when compared to non-MUA residence. Distance to clinic exhibited a dose-response relationship such that patients who lived farthest from the clinics were most likely to have telemedicine visits. Compared to residence within 5 miles of a clinic, patients who were 5-10 miles away from a clinic had $12 \%$ higher odds of a telemedicine visit $(\mathrm{OR}=1.12, p<0.001)$; patients $10-20$ miles away had 20\% higher odds of a telemedicine visit (OR $=1.20, p<$ 0.001 ); patients $20-50$ miles away had $35 \%$ higher odds of a telemedicine visit $(\mathrm{OR}=1.35, p<0.001)$; and patients more than than 50 miles away had 2.04 times higher odds of a telemedicine visit $(\mathrm{OR}=2.04, p<0.001)$.

Table III also reports the intraclass correlations for the threelevel nested model. Conditional on the fixed-effects covariates, patient random effects compose $37 \%$ (ICC: 0.37 ) of the total residual variance, while patient and clinic random effects compose 59\% (ICC: 0.59 ) of the total residual variance.

\section{Cohort subanalyses}

Results of the race/ethnicity-stratified models to examine correlates of telemedicine use in African American and Hispanics cohorts separately are presented in Table IV. Among Hispanics, compared to patients who lived $<5$ miles away from the clinic, there was a higher likelihood of telemedicine use among patients who lived $5-10$ miles $(\mathrm{OR}=1.14 ; p<0.001)$, $10-20$ miles $(\mathrm{OR}=1.14 ; p<0.001), 20-50$ miles $(1.25 ; p<$ $0.001)$, and $\geq 50$ miles away from the clinic $(\mathrm{OR}=1.73 ; p<$ 0.001). Living in an MUA was also associated with a higher likelihood of telemedicine use $(\mathrm{OR}=1.18 ; p$ value $<$ $0.001)$ compared to living in a non-MUA. Negative correlates of telemedicine use in this cohort included having Medicaid insurance $(\mathrm{OR}=0.84, p=0.014)$, being uninsured $(\mathrm{OR}=0.82$, $p=0.011)$, being less than 18 years $(\mathrm{OR}=0.36, p<$ $0.001)$, being over 65 years of age $(\mathrm{OR}=0.63, p=0.003)$. There was no statistically significant association between metropolitan residence and telemedicine use. 
Table I Descriptive Statistics of Patient Characteristics By Visit Type

\begin{tabular}{|c|c|c|c|c|c|c|c|}
\hline \multirow[t]{3}{*}{ Variables } & \multirow{2}{*}{\multicolumn{2}{|c|}{$\begin{array}{l}\text { Total Visits } \\
(n=233,302)\end{array}$}} & \multicolumn{5}{|c|}{ Visit type } \\
\hline & & & \multicolumn{2}{|c|}{$\begin{array}{l}\text { Face-to-face visit } \\
(n=176,971)\end{array}$} & \multicolumn{2}{|c|}{$\begin{array}{l}\text { Telemedicine } \\
\text { visit }(n=56,331)\end{array}$} & \multirow[t]{2}{*}{$p$ value } \\
\hline & $N$ & $(\%)$ & $N$ & $(\%)$ & $N$ & $(\%)$ & \\
\hline Race & & & & & & & $<0.001$ \\
\hline White & 181,032 & $(77.6)$ & 137,507 & $(76.0)$ & 43,525 & $(24.0)$ & \\
\hline Black /African American & 35,165 & $(15.1)$ & 26,483 & $(75.3)$ & 8,682 & $(24.7)$ & \\
\hline Asian & 7,456 & $(3.2)$ & 5,689 & $(76.3)$ & 1,767 & $(23.7)$ & \\
\hline American Indian / Alaska Native, Other Pacific Islander & 3,362 & $(1.4)$ & 2,679 & $(79.7)$ & 683 & $(20.3)$ & \\
\hline Mixed race & 6,287 & $(2.7)$ & 4,613 & $(73.4)$ & 1,674 & $(26.6)$ & \\
\hline Ethnicity & & & & & & & $<0.001$ \\
\hline Non-Hispanic & 121,536 & $(52.1)$ & 86,624 & $(71.3)$ & 34,912 & $(28.7)$ & \\
\hline Hispanic & 111,766 & $(47.9)$ & 90,347 & $(80.8)$ & 21,419 & $(19.2)$ & \\
\hline Age & & & & & & & $<0.001$ \\
\hline$<18$ & 91,424 & $(39.2)$ & 72,639 & $(79.5)$ & 18,785 & $(20.5)$ & \\
\hline $18-64$ & 131,300 & $(56.3)$ & 96,708 & $(73.7)$ & 34,592 & $(26.3)$ & \\
\hline$>=65$ & 10,578 & $(4.5)$ & 7,624 & (72.1) & 2,954 & $(27.9)$ & \\
\hline Gender & & & & & & & $<0.001$ \\
\hline Female & 153,217 & $(65.7)$ & 115,446 & $(75.4)$ & 37,771 & $(24.7)$ & \\
\hline Male & 80,085 & $(34.3)$ & 61,525 & $(76.8)$ & 18,560 & $(23.1)$ & \\
\hline Insurance coverage & & & & & & & $<0.001$ \\
\hline Private insurance & 40,235 & (17.4) & 28,078 & $(69.8)$ & 12,157 & $(30.2)$ & \\
\hline Medicare & 13,628 & $(5.9)$ & 8,849 & (64.9) & 4,779 & $(35.1)$ & \\
\hline Medicaid & 100,551 & (43.4) & 79,562 & $(79.1)$ & 20,989 & $(20.9)$ & \\
\hline Uninsured & 77,242 & $(33.3)$ & 58,883 & $(76.2)$ & 18,359 & $(23.8)$ & \\
\hline Acute-visit flag & & & & & & & $<0.001$ \\
\hline Nonacute visit & 166,455 & $(71.3)$ & 123,416 & $(74.1)$ & 43,039 & $(25.9)$ & \\
\hline Acute visit & 66,847 & $(28.7)$ & 53,555 & $(80.1)$ & 13,292 & $(19.9)$ & \\
\hline Geographic characteristics of patient residence & & & & & & & \\
\hline Metropolitan status & & & & & & & $<0.001$ \\
\hline Nonmetropolitan & 8,668 & $(3.7)$ & 6,834 & $(78.8)$ & 1,834 & $(21.2)$ & \\
\hline Metropolitan & 224,506 & (96.3) & 170,059 & $(75.7)$ & 54,447 & $(24.3)$ & \\
\hline MUA status & & & & & & & 0.921 \\
\hline Non-MUA & 117,423 & $(50.4)$ & 89,091 & $(75.9)$ & 28,332 & $(24.1)$ & \\
\hline MUA & 115,751 & $(49.6)$ & 87,802 & $(75.9)$ & 27,949 & $(24.1)$ & \\
\hline Distance from FQHC clinic & & & & & & & $<0.001$ \\
\hline$<5$ miles & 63,748 & $(27.3)$ & 49,123 & $(77.1)$ & 14,625 & $(22.9)$ & \\
\hline $5-10$ miles & 60,169 & $(25.8)$ & 46,011 & $(76.5)$ & 14,158 & $(23.5)$ & \\
\hline $10.01-20$ miles & 65,499 & $(28.1)$ & 49,651 & $(75.8)$ & 15,848 & $(24.2)$ & \\
\hline $20.01-50$ miles & 38,117 & $(16.3)$ & 28,436 & $(74.6)$ & 9,681 & $(25.4)$ & \\
\hline$>50$ miles & 5,769 & $(2.5)$ & 3,750 & $(65.0)$ & 2,019 & (34.9) & \\
\hline
\end{tabular}

Among Blacks / African Americans, the dose-response relationship between distance from clinic and telemedicine use was also observed with increased likelihood of telemedicine use among those living 10-20 miles (OR $=1.22, p<$ $0.001), 20-50$ miles $(\mathrm{OR}=1.28, p<0.001)$ and $\geq 50$ miles $(\mathrm{OR}=2.31, p<0.001)$ compared to those living $<5$ miles away from the clinic. In contrast to the finding among Hispanics, living 5-10 miles away from the clinic was not significantly associated with telemedicine use. There was no significant association between MUA residence and telemedicine use. Similar to the findings among Hispanics, Blacks / African Americans who were Medicaid-insured $(\mathrm{OR}=0.84, p<$ $0.001)$, uninsured $(\mathrm{OR}=0.82, p<0.001)$, younger than 18 years $(\mathrm{OR}=0.36, p<0.001)$, and 65 years or older $(\mathrm{OR}=$ $0.63, p<0.001)$ were less likely to use telemedicine. In contrast to the findings among Hispanics, there was no statistically significant association between male sex, MUA residence, and acute visit. Intraclass correlation coefficients (ICC) revealed weak-to-moderate correlations within patient and clinic random effects. Among Hispanic patients, patient

Table II Telemedicine Use by Service Line over Time (March-November 2020)

\begin{tabular}{|c|c|c|c|c|c|}
\hline \multirow[t]{2}{*}{ Month } & \multicolumn{5}{|c|}{ Telehealth use (\%) by service line } \\
\hline & Family practice & Mental Health & Obstetrics and Gynecology & Pediatrics & Senior Care \\
\hline March 2020 & 0 & 11.1 & 0 & 0 & 0 \\
\hline April 2020 & 32.4 & 70.2 & 5.0 & 13.8 & 58.9 \\
\hline May 2020 & 36.5 & 67.9 & 5.5 & 14.0 & 43.4 \\
\hline June 2020 & 33.9 & 56.9 & 4.4 & 14.6 & 35.1 \\
\hline July 2020 & 33.5 & 57.8 & 3.8 & 16.2 & 45.8 \\
\hline August 2020 & 29.3 & 56.9 & 3.4 & 12.3 & 31.2 \\
\hline September 2020 & 30.6 & 56.9 & 3.1 & 15.1 & 18.9 \\
\hline October 2020 & 28.5 & 53.5 & 3.0 & 11.4 & 23.8 \\
\hline November 2020 & 29.8 & 54.1 & 2.4 & 11.3 & 22.0 \\
\hline
\end{tabular}


Table III Mixed-Effects Logistic Regression Modeling of the Relationship Between Telemedicine and Patient Characteristics $(n=$ 231,527)

\begin{tabular}{|c|c|c|c|c|c|}
\hline \multirow[t]{2}{*}{ Variables } & \multicolumn{5}{|c|}{ MV-adjusted OR } \\
\hline & OR & \multicolumn{3}{|c|}{$95 \% \mathrm{CI}$} & $\begin{array}{l}p \\
\text { value }\end{array}$ \\
\hline \multicolumn{6}{|l|}{ Race } \\
\hline White & Ref. & & & & \\
\hline Black /African & 0.65 & 0.61 & - & 0.69 & $<0.001$ \\
\hline \multicolumn{6}{|l|}{ American } \\
\hline Asian & 0.58 & 0.52 & - & 0.65 & $<0.001$ \\
\hline American Indian / & 0.82 & 0.70 & & 0.98 & 0.025 \\
\hline \multicolumn{6}{|l|}{ Alaska Native, Other } \\
\hline \multicolumn{6}{|l|}{ Pacific Islander } \\
\hline Mixed race & 0.99 & 0.88 & - & 1.12 & 0.881 \\
\hline \multicolumn{6}{|l|}{ Ethnicity } \\
\hline Non-Hispanic & Ref. & & & & \\
\hline Hispanic & 0.49 & 0.47 & - & 0.51 & $<0.001$ \\
\hline \multicolumn{6}{|l|}{ Age } \\
\hline $18-64$ & Ref. & & & & \\
\hline$<18$ & 0.38 & 0.36 & - & 0.41 & $<0.001$ \\
\hline$>=65$ & 0.82 & 0.74 & - & 0.91 & $<0.001$ \\
\hline \multicolumn{6}{|l|}{ Gender } \\
\hline Female & Ref. & & & & \\
\hline Male & 0.85 & 0.81 & - & 0.88 & $<0.001$ \\
\hline \multicolumn{6}{|l|}{ Insurance coverage } \\
\hline Private insurance & Ref. & & & & \\
\hline Medicare & 1.96 & 0.87 & - & 1.06 & 0.442 \\
\hline Medicaid & 0.76 & 0.72 & _- & 0.81 & $<0.001$ \\
\hline Uninsured & 0.67 & 0.63 & - & 0.71 & $<0.001$ \\
\hline \multicolumn{6}{|l|}{ Metropolitan status } \\
\hline Nonmetropolitan & Ref. & & & & \\
\hline Metropolitan & 1.17 & 1.02 & - & 1.33 & 0.023 \\
\hline \multicolumn{6}{|l|}{ MUA status } \\
\hline Non-MUA & Ref. & & & & \\
\hline MUA & 1.12 & 1.07 & - & 1.18 & $<0.001$ \\
\hline \multicolumn{6}{|l|}{ Acute-visit flag } \\
\hline Nonacute visit & Ref. & & & & \\
\hline Acute visit & 0.89 & 0.86 & - & 0.92 & $<0.001$ \\
\hline \multicolumn{6}{|c|}{ Distance from $\mathrm{FQHC}$ clinic } \\
\hline$<5$ miles & Ref. & & & & \\
\hline 5-10 miles & 1.12 & 1.06 & - & 1.18 & $<0.001$ \\
\hline $10-20$ miles & 1.20 & 1.14 & - & 1.27 & $<0.001$ \\
\hline 20-50 miles & 1.35 & 1.27 & - & 1.44 & $<0.001$ \\
\hline$\geq 50$ miles & 2.04 & 1.78 & - & 2.33 & $<0.001$ \\
\hline \multicolumn{6}{|c|}{ Intraclass correlation coefficient for clinic and patient random effects } \\
\hline & $\begin{array}{l}\text { Intraclass } \\
\text { correlation }\end{array}$ & $95 \%$ & CI & & $\begin{array}{l}\text { Std } \\
\text { err }\end{array}$ \\
\hline Patient ID & 0.37 & 0.25 & - & 0.46 & 0.042 \\
\hline Patient ID / clinic & 0.59 & 0.53 & - & 0.65 & 0.030 \\
\hline location & & & & & \\
\hline
\end{tabular}

random effect ICC was 0.35 , while patient and clinic ICC was 0.56. Comparatively among Black / African American patients, patient random effect ICC was 0.39 , while patient and clinic random effect ICC was 0.57 .

\section{DISCUSSION}

This study examined disparities in telemedicine use in FQHC clinics during the coronavirus pandemic. In this large multiclinic study, we found that compared to Whites, minority-race / ethnic populations, including African Americans, Hispanics, American Indians / Alaska Natives and other Pacific Islanders, and Asians, were less likely to have a telemedicine visit. Individuals younger than 18 years and older adults were less likely to have a telemedicine visit when compared to non-elderly adults, as were those covered
Table IV Correlates of Telemedicine Use in African Americans and Hispanics/Latinx

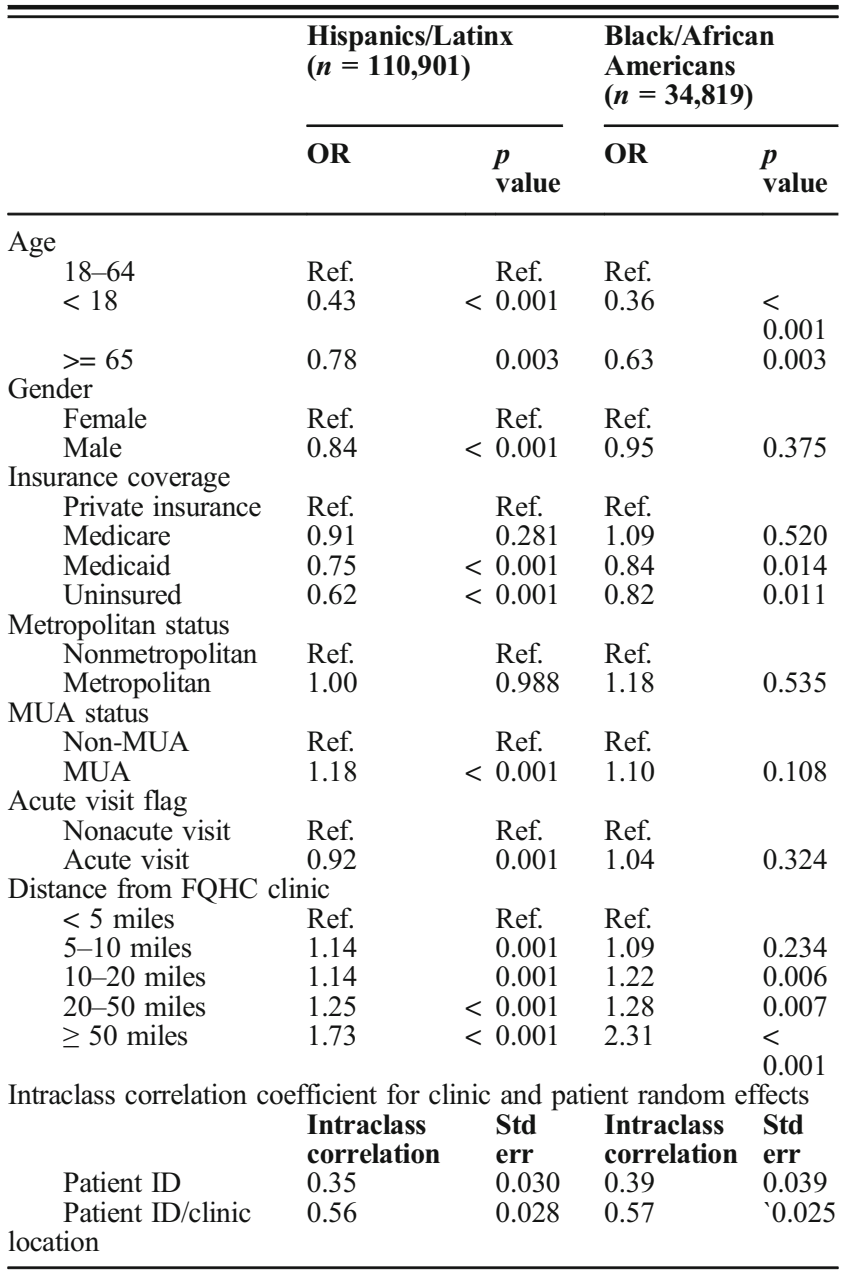

under Medicaid coverage, or uninsured. Residence in a metropolitan area and non-acute visits were associated with increased telemedicine use. We also found a dose-response relationship between distance from clinic and telemedicine use with increasing likelihood of telemedicine use with increasing distance from the clinic. This finding was consistent for the general sample, as well as on stratified analyses that focused onAfrican Americans and Hispanics, separately.

Our findings of persistent racial/ethnic disparities in telemedicine use are consistent with previous studies that have reported a lower likelihood of health information technology use among racial/ethnic minorities in the prepandemic and pandemic era. For example, Mitchell et al. (2019) found Black / African American and Hispanic adults to face a decreased likelihood of using technology for health-related purposes. ${ }^{12}$ Fischer et al. (2020) revealed Blacks / African Americans as less likely to express willingness to use telemedicine services. ${ }^{13}$ Another study found Asians to experience $42 \%$ lower odds of a telemedicine visit compared to Whites and found Blacks / African Americans 8\% less likely. ${ }^{14}$ Evidence of racial disparities in telemedicine 
utilization underscores the importance of addressing digital health equity to improve health care access.

Health information tools such as telemedicine hold promise for reducing health and health care disparities and ultimately improving population health. ${ }^{15}$ A systematicreview reported that telemedicine is associated with improved chronic disease management. ${ }^{16,17}$ Another systematic literature review reported better glycemic index and systolic blood pressure control among patients using telemedicine compared to usual care. ${ }^{18}$ These findings underscore the role of telemedicine in improving clinical outcomes and potentially reducing health disparities. Our finding of persistent racial/ethnic disparities suggests that the promise of the positive impact of telemedicine on health care use and health outcomes could elude underserved populations. It is therefore critical to identify factors that adversely affect telemedicine use among racial/ethnic minorities as this could aid strategies to increase telemedicine adoption among these subpopulations. Further studies are also needed to elucidate how factors that have been previously associated with decreased health information technology use or health care utilization, such as broadband access, ${ }^{19}$ limited health literacy, ${ }^{20}$ and patient preference, could limit the adoption of telemedicine among racial/ethnic minorities.

In our study, we found that telemedicine utilization differed significantly by type of patient insurance coverage. These variations persisted on stratified analyses restricted to African Americans and Hispanics, with Medicaid-insured patientsand uninsured patients having a lower likelihood of telemedicine use. This is in contrast with other studies that have founda higher likelihood of telemedicine use among patients enrolled in Medicaid compared to those privately insured. ${ }^{21,22}$ Nevertheless, Wegermann et al. (2021) also noted that Medicaid insurance is associated with a higher likelihood of incomplete telephone visits. ${ }^{22}$ This difference between our study and the previous studies could be attributed to different study populations - our study focused on patients receiving care at FQHCs, while these earlier studies focused on patients receiving care at academic medical centers.

Demographic factors associated with reduced likelihood of telemedicine use in our study include being younger than 18 years or older than 64 years for the general sample, as well as among Hispanics and African Americans. Male sex was also associated with decreased likelihood of telemedicine use among African American but not Hispanics. The latter observed trend aligns with previous studies that have suggested that older adults are overall less likely to have telemedicine visits, perhaps due to decreased technological knowledge. ${ }^{21}$ Thus, interventions that target these subgroups have potential for increasing telemedicine use.

Our findings also portray that telemedicine use differed by acute vs. nonacute visit type. While those with acute health care visits were less likely to have a telemedicine visit, nonacute visits were positively associated with telemedicine use in the Hispanic cohort, but not in the Black / African American cohort. Indeed, one of the limitations of telemedicine is that it is often not appropriate for patients who require emergent medical care, as certain necessary procedures or physical examinations cannot be performed in a remote setting. Some have suggested the use of telemedicine in acute care settings, however, especially in the face of the COVID-19 pandemic, to reduce the burden on emergency departments and in-patient services, Lin et al. (2020) argued that telemedicine in the acute care setting reduces time and exposure to infectious patients and offers the option of "forward triage," which is triaging patients before they arrive to the clinic. ${ }^{23}$

Geographical characteristics, such as nonmetropolitan residence, have been associated with suboptimal health care use, thus providing an opportunity for telemedicine intervention. In this study, those living in metropolitan areas had a significantly increased likelihood of having a telemedicine visit when compared to those in nonmetropolitan areas, although this relationship was no longer statistically significant on stratification by race/ethnicity. This is in line with previous evidence that suggests a reduced likelihood in telemedicine use for rural and nonmetropolitan residents. ${ }^{21}$ Rural residents face greater health care-related travel burdens when pursuing access to their medical care, ${ }^{24}$ which provides an opportunity for telemedicine interventions to address these barriers. However, barriers to telemedicine adoption, including limited availability of broadband internet, ${ }^{25}$ could limit telemedicine use among rural and nonmetropolitan residents. A previous study associated the digital divide/gap in internet access between rural and urban neighborhoods with socioeconomic differences in income and level of education. ${ }^{26}$

Finally, our findings highlight the impact of other geographic characteristics on health care utilization via telemedicine services. We found MUA residence to be associated with increased likelihood of telemedicine use compared to nonMUA residence, and upon stratification analyses, this relationship persisted for Hispanics but not for African Americans. We also observed a dose-response relationship between distance from a clinic and telemedicine use such that that patients living 20-50 miles away from the FQHC clinic had 35\% greater odds of having a telemedicine visit when compared to those living within 5 miles of a clinic. Those living more than 50 miles away had $104 \%$ greater odds of telemedicine use, and this relationship was even more marked among African Americans on stratified analyses (131\%). Previous studies have found that MUA residence or increased distance from clinic isassociated with a decreased likelihood of health care use. ${ }^{27}$ Therefore, the findings in the index study imply that telemedicine offers immense potential for improving access to care, especially those who face geographic or transportation barriers to healthcare.

This study is not without its limitations. Our analyses use data from $\mathrm{FQHC}$ clinics, and therefore, these findings may not be representative of other types of clinic across the country. There are also various challenges in widespread telemedicine adoption for FQHCs that are not fully addressed in this study, 
such as usability, perceived privacy or security threats, ${ }^{28}$ or patient preference. This study also occurred during the initial/ peak phase of the COVID-19 pandemic that was characterized by significant disruptions to the health care system - this limits the generalizability of our study findings to a postpandemic period. Nevertheless, our study also has several strengths. The use of data from a large FQHC with 55 clinics enables us to generalize our findings to a large patient population. Our use of recent data also provides insight into disparities in telemedicine adoption in this COVID-19 era.

In conclusion, while telemedicine offers immense potential in reducing gaps in access to health care, our findings suggest a residual lag in adoption by minority populations, which could exacerbate long-standing disparities. Recognizing determinants of disparate telemedicine adoption provides an opportunity for targeted interventions to increase telemedicine use among racial/ethnic minorities.

Corresponding Author: Omolola E. Adepoju, $\mathrm{PhD}, \mathrm{MPH}$; University of Houston, College of Medicine, 4849 Calhoun Road, Bldg 2, Houston, TX 77204, USA (e-mail: oadepoju@central.uh.edu).

\section{Declarations:}

Conflict of Interest: The authors declare that they do not have a conflict of interest.

\section{REFERENCES}

1. Buchmueller TC, et al. Effect of the Affordable Care Act on racial and ethnic disparities in health insurance coverage. Am J Public Health. 2016;106(8): 1416-1421.

2. Buchmueller TC, Levy HG. The ACA's Impact On Racial And Ethnic Disparities In Health Insurance Coverage And Access To Care: An examination of how the insurance coverage expansions of the Affordable Care Act have affected disparities related to race and ethnicity. Health Aff. 2020;39(3):395-402.

3. Adepoju, O.E., M.A. Preston, and G. Gonzales, Health care disparities in the post-Affordable Care Act era. Am J Public Health. 2015;105(S5):S665-S667.

4. Artiga S, Orgera K, Pham O. Disparities in health and health care: Five key questions and answers. Kaiser Family Foundation. 2020. Accessed on July 22, 2021 at https://files.kff.org/attachment/Issue-Brief-Disparities-in-Health-and-Health-Care-Five-Key-Questions-and-Answers.

5. Adepoju O, et al. COVID-19 and Telehealth Operations in Texas Primary Care Clinics: Disparities in Medically Underserved Area Clinics. J Health Care Poor Underserved. 2021;32(2):948-957.

6. Coronavirus Aid, Relief, and Economic Security Act, S. 3548, US 116th Congress, 2d Sess.

7. Brown EJ, et al. Racial disparities in geographic access to primary care in Philadelphia. Health Aff. 2016;35(8):1374-1381

8. Syed ST, Gerber BS, Sharp LK. Traveling towards disease: transportation barriers to health care access. J Community Health. 2013;38(5):976-993.
9. Perrin A. Demographics of Internet and Home Broadband Usage in the United States | Pew Research 2018. Access on July 22, 2021 at https:// www.pewresearch.org/internet/fact-sheet/internet-broadband/.

10. Waldman J. COVID-19 Deepens a Digital Divide that has long impacted low-income, Black and Hispanic, Tribal and Rural, and older households, according to recent reports (Digital Divide Blog Series: Part 1). Connecting Justice Communities. 2020.

11. Reicher S, Sela T, Toren O. Using telemedicine during the Covid-19 pandemic: Attitudes of adult health care consumers in Israel. Front Public Health., 2021. 9:3553.

12. Mitchell UA, et al. The digital divide in health-related technology use: The significance of race/ethnicity. Gerontologist. 2019;59(1):6-14

13. Fischer SH, et al. Prevalence and characteristics of Telehealth utilization in the United States. JAMA Netw Open. 2020;3(10):e2022302-e2022302.

14. Jaffe DH, et al. Health Inequalities in the Use of Telehealth in the United States in the Lens of COVID-19. Popul Health Manag. 2020;23(5):368377.

15. Pérez-Stable EJ, Jean-Francois B, Aklin CF. Leveraging advances in technology to promote health equity. Med Care. 2019;57:S101-S103.

16. Beratarrechea A, et al. The impact of mobile health interventions on chronic disease outcomes in developing countries: a systematic review. Telemed e-Health., 2014;20(1):75-82.

17. Flodgren $\mathrm{G}$, et al. Interactive telemedicine: effects on professional practice and health care outcomes. Cochrane Database Syst Rev. 2015;2015(9):CD002098.

18. Wu C, et al. Evaluation of the clinical outcomes of telehealth for managing diabetes: a PRISMA-compliant meta-analysis. Medicine. 2018. 97(43):e12962.

19. Jain V et al. Racial and Geographic Disparities in Internet Use in the US Among Patients With Hypertension or Diabetes: Implications for Telehealth in the Era of COVID-19. Diabetes Care. 2021;44(1):e15-e17.

20. Eneanya ND, et al. Health literacy and education as mediators of racial disparities in patient activation within an elderly patient cohort. J Health Care Poor Underserved. 2016. 27(3): p. 1427.

21. Pierce RP, Stevermer JJ. Disparities in use of telehealth at the onset of the COVID-19 public health emergency. J Telemed Telecare. $2020 ; 1357633 \times 20963893$. https://doi.org/10.1177/ 1357633 X20963893.

22. Wegermann K, Wilder JM, Parish A, Niedzwiecki D, Gellad ZF, Muir AJ, Patel YA. Racial and socioeconomic disparities in utilization of telehealth in patients with liver disease during COVID-19. Digestive diseases and sciences. 2021;28:1-7.

23. Lin $Z$, et al. Telemedicine in the acute care setting during the COVID-19 pandemic. Intern Emerg Med., 2020;15(8):1591-1593.

24. Probst JC, et al. Effects of residence and race on burden of travel for care: cross sectional analysis of the 2001 US National Household Travel Survey. BMC Health Serv Res. 2007;7(1):1-13.

25. Cortelyou-Ward K, et al. Navigating the Digital Divide: Barriers to Telehealth in Rural Areas. J Health Care Poor Underserved. 2020;31(4): 1546-1556.

26. Whitacre BE, Mills BF. Infrastructure and the rural-urban divide in high-speed residential Internet access. Int Reg Sci Rev. 2007;30(3):249273.

27. Jackson BM, et al. Unmet primary care needs in diabetic patients with multimorbidity in a medically underserved area. Health Serv Res Manag Epidemiol. 2017;4:2333392817702760.

28. Menachemi N, Burke DE, Ayers DJ. Factors affecting the adoption of telemedicine-a multiple adopter perspective. J Med Syst. 2004;28(6):617-632

Publisher's Note: Springer Nature remains neutral with regard to jurisdictional claims in published maps and institutional affiliations. 